22. Otnaess, A. B. and Orstavik, I.: Effect of fractions of Ethiopian and Norwegian colostrum on rotavirus and escherichia coli heat-labile enterotoxin. Infect. Immun., 33: 459 (1981).

23. Quinnan. G. V. and Manischewitz, J. E.: The role of natural killer cells and antibody dependent cell-mediated cytotoxicity during murine cytomegalovirus infection. J. Exp. Med., 150:1549 (1979).

24. Santoli, D., Trinchieri, G., and Koprowski. H.: Cell-mediated cytotoxicity against virus-infected target cells in humans. II. Interferon induction and activation of natural killer cells. J. Immunol., 121: 532 (1978).

25. Sonza, S. and Holmes, I. H.: Coproantibody response to rotavirus infection. Med. J. Australia, 2: $496(1980)$

26. Toterdell, B. M., Chrystie, I. L., and Banatvala, J. E.: Cord blood and breastmilk antibodies in neonatal rotavirus infection. Br. Med. J. 280:828 (1980).

27. Trincheri, G. and Santoli, D.: Antiviral activity induced by culturing lymphocytes with tumor-derived or virus-transformed cells. Enhancement of natural killer cell activity by interferon and antagonistic inhibition of susceptibility of target cells to lysis. J. Exp. Med. 147: 1314 (1978).

28. Weaver, E. A., Goldblum, R. M.. Davis. C. P., and Goldman, A. S.: Enhanced immunoglobulin A release from human colostral cells during phagocytosis. Infect. Immun.. 34: 498 (1981).

29. Welsh, R. M.: Cytotoxic cells induced during lymphocytic choriomeningitis virus infection of mice. I. Characterization of natural killer cell induction. J. Exp. Med. 148: 163 (1978).

30. Yolken, R. H., Wyatt, R. G., Mata, L., Urrutia, J. J., Garcia, B., Chanock, R. M., and Kapikian, A. Z.: Secretory antibody directed against rotavirus in human milk-measurement by means of enzyme-linked immunosorbent assay. J. Pediatr. 93:916 (1978).

31. We would like to acknowledge the technical assistance of Lian Sim Loo, Mary Neil and Dixie Baez, and the secretarial assistance of Ms. Shirley Washington. This work was supported by grant PO $1 \mathrm{HD} 13021$ and contract no. AI 32506 from the National Institutes of Health.

32. Requests for reprints should be addressed to: Dr. Steve Kohl, Program in Infectious Diseases and Clinical Microbiology, 6431 Fannin Street, Houston. Texas 77030.

33. Received for publication August 5, 1982.

34. Accepted for publication March 18, 1983.

\title{
Behavioral and Cardiac Rhythmicity during Mother-Father-Stranger Infant Social Interaction
}

\author{
MICHAEL W. YOGMAN, ${ }^{(25)}$ BARRY M. LESTER, AND JOEL HOFFMAN \\ Department of Pediatrics, Harvard Medical School, and Child Development Unit, Children's Hospital Medical \\ Center, Boston, Massachusetts, USA
}

\begin{abstract}
Summary
In an effort to understand the temporal organization of infant physiologic and behavioral systems during social interaction with adults, spectral and cross-spectral analyses of infant heart rate and mother-father-stranger-infant behavioral data are described herein for a 3-month-old infant during face-to-face social interaction with her parents and a stranger. This infant's heart rate rhythms were stronger during social interaction with both mother and father than with a stranger. Infant behavioral rhythms were associated with (high coherence) the parents' behavioral rhythms, but not with those of the stranger. Furthermore, infant heart rate and infant behavioral rhythms showed high coherence with all three adults. Although these findings are preliminary, based on a case study and need replication, they generate intriguing hypotheses. This technique of studying the rhythmicity of infant social interaction with spectral analysis suggests that the 3-month-old infant's behavior and heart rate are synchronized during interaction with all three adults; however, the infant and adult behavioral rhythms are synchronous only with mother and father and not with an unfamiliar stranger.
\end{abstract}

Research on biologic rhythms suggests that a wide range of rhythmic phenomena in the human organism have a biologic basis deriving from the periodic transmission of impulses from a specialized area of the central nervous system, a biologic clock (13). The development of an adaptive fit between the rhythms of the newborn and the extrinsic rhythmicity of the environment is an evolving area of research. For example, Condon and Sander (5) have suggested that the human neonate synchronizes its body motions to the phonemes of adult speakers. Recent work has emphasized the contributions made by caregivers to facilitate the temporal organization of the infant's various functions such as sleeping and feeding, which become coordinated within the baby and linked with the regular periodicities of the physical and social environment (15). Although the presence of rhythmicity in both behavioral and physiologic systems in the infant may have a biologic basis, the relationship between behavioral and physiologic rhythms may be influenced by the sensitivity of the infant's caregiving environment. One might also expect that the rhythmic structure of the infant's behavior would vary if the feedback provided by a strange adult differs from that of the parent during social play.

The concept of rhythmicity in infant-adult social interactions has long been of interest to scientists studying the structure, development, and biologic basis of social communication $(2,16$, 19). Studies of mother-infant interaction $(18,1,3,11)$ assume that rhythmicity is part of the underlying structure of the interaction and are attempting to uncover the rules that govern the relationship between the behavior of the two participants. When the two partners cycle together, the interaction is described as synchronous and the degree of synchrony has been suggested as a measure of the quality of the interaction $(3,4)$.

Descriptive studies of infant behavior with other adults suggest that infants interact differently with mothers than with fathers $(7,22,23)$ or with strangers $(6)$, although the rhythmic structure of these interactions has not been studied systematically or quantified. With mothers and infants, one of the patterns described has been the build-up and withdrawal of attention. Infants and mothers appeared to increase and decrease their behavioral attention and arousal in a periodic, cyclical fashion (2) that may reflect a relationship between infant behavioral and physiologic rhythms. The authors suggested that both the infant's behavioral state or arousal and his physiologic systems are related 
and operate under rules of feedback and control similar to any homeostatic system.

The behaviors involved in social interactions shift during short intervals enabling investigators of mother-infant and father-infant interaction to time-sample observations and generate data in a time series $(21,23)$. Until recently, techniques for the study of rhythmicity have not been available; therefore, most attempts to study social interaction have been descriptive or used correlations, sequential or lag analyses, or conditional probabilities to look at associations among behaviors $(1,21)$. Behavioral and physiologic phenomena are likely to be composed of more than one rhythm, and therefore frequency domain, rather than time domain procedures, are required to separate these complex rhythms and more adequately describe their features. Gottman (8) has shown that the frequency domain techniques of spectral analysis can be applied to the study of rhythmicity in human behavior and social interaction in individual dyads. The generation of two simultaneous time series recorded from dyadic interaction has enabled Gottman $(8,9)$ to study the relationships between the behavioral cycling during social interaction, between two adult partners and between mothers and infants. With this technique, Gottman operationalizes measures of synchrony during social interaction. Spectral analysis has also been used to study social interaction between term and preterm infant-mother dyads (12) and to assess the rhythmicity of heart rate and the shared rhythmicity or coupling of cardiorespiratory rhythmicity across an entire band of frequencies (14).

This paper is a first attempt to link rhythmicity in behavior and physiology. We have used this technique of spectral analysis to investigate rhythmicity by studying simultaneous infant behavioral and physiologic (heart rate) changes during dyadic social interaction between a 3-month-old infant and her father, mother, and a stranger. Specifically, we asked ( $I$ ) does rhythmicity in infant behavior differ during interactions with mother, father, and stranger? (2) what is the relationship between infant behavioral and physiologic rhythms? (3) does rhythmicity in infant physiology differ during interactions with mother, father, and stranger, and (4) does the association or synchrony among infant and adult cycles differ depending on the adult?

\section{MATERIALS AND METHODS}

The subject for this study was a 98-day-old, healthy female infant with her mother and father. The infant was full term, weighed $3040 \mathrm{~g}$ at birth, was delivered vaginally after an uncomplicated pregnancy, and had 1- and 5-min Apgars of 9 and 10. Mother was the primary caretaker and the stranger was a woman without children.

The infant was seen in a laboratory for 2 min of face-to-face interaction, first with mother, then with stranger, and then with father. Each episode was separated by $30 \mathrm{sec}$ of infant alone. This method (3) involved seating the infant in a seat placed on a table surrounded by curtains, instructing the adult to enter, sit in front of the infant and play without using toys and without removing the infant from the seat. Electrocardiographic leads were attached to the infant's limbs and second-by-second digital heart rate was displayed on a Hewlett Packard Model 78203A monitor. Heart rate was calculated from a moving average across every three beats. One video camera focused on the infant, the other on the adult. The two images appeared simultaneously as a split screen display on a television monitor, which showed a single frontal view of adult and infant along with infant heart rate depicted on the cardiotachometer in the lower portion of the screen along with a digital time display. Sound was simultaneously recorded.

The videotape was analyzed using a microbehavioral secondby-second scoring system which generated two, 120-sec continuous time series, describing the behaviors displayed by each participant over the 2-min interaction. The first step in the coding procedure was to score for each second, four behaviors for the infant (gaze pattern, limb movement, facial expression, and vocalization) and five behaviors for the adult (gaze pattern, body position, facial expression, vocalization, and touching pattern) according to a previously established scoring system (6). Tapes were coded in slow motion as the occurrence of each behavior in a 1 -sec time period. Infant behaviors were scored with the adult side of the split screen monitor covered. Reliability was evaluated in independent scoring of videotapes. Two independent observers agreed greater than $85 \%$ of the time for infant behaviors, and greater than $80 \%$ of the time for adult behaviors.

Based on previous work, which suggested that the interactive messages and communicative meaning were carried in clusters of substitutable behaviors rather than discrete behaviors (21), in a second step, we clustered the coded discrete behaviors. We used a priori decision rules to cluster the coded behaviors into more meaningful units of analysis called monadic phases. This system was developed by Tronick et al. $(20,21)$ and modified for this study by Yogman $(22,23)$. Each second-by-second cluster of behavior was translated into one of the following monadic phases: talk, play, set, elicit, monitor, avert, and protest/avoid. The choice of phases and rules for clustering were guided by the expectation that each phase would convey an affective message from one partner to the other (21); therefore, we derived one monadic phase on a 7 point ordinal scale, representing a continuum of negative to neutral to positive affect, for each second of interaction separately for adult and infant. This yielded a continuous time series consisting of 120 data points for each individual. This behavioral data or heart rate was used as the input data for spectral analysis performed separately on each time series. Gottman and Ringland (9) have shown the applicability of spectral analysis to dichotomous or ordinal social interaction data for time series of at least 100 data points. We generated nine separate 120 -sec time series; one each for infant heart rate, infant behavior, and adult behavior when the infant was interacting with each of the three adults.

Spectral analysis is a frequency (cycles per sec) domain procedure that decomposes a complex waveform into its constituent sine waves. In the frequency domain, periodicity is expressed as either cycles per second $(\mathrm{Hz})$ or the duration (period) of each cycle. Cross-spectral analysis analagous to cross-correlation was computed between infant and adult behavior and between infant heart rate and behavior to derive the coherence spectrum. Coherence gives the proportion of shared variance for the two series at each frequency and is analogous to the square of the correlation. Coherence may be considered a measure of the association or synchronicity between two rhythms.

\section{RESULTS}

The data from the mother-infant episode are shown in Figure 1. The sequence of phases with adult and infant superimposed is shown with infant heart rate depicted above the monadic phase data. To meet the assumption of stationarity for spectral analysis, linear trends were removed before the analyses. The SAS SPECTRA program (17) was used to first fit sine and cosine functions to the stationary time series using the Fast Fourier Transform. The periodogram generated by this analysis was smoothed using a triangular window to yield spectral densities, which estimate the variance accounted for by each frequency $(\mathrm{Hz})$. This method of partitioning of the variance is similar to the partitioning of variance into sums of squares in traditional analyses of variance. The spectral densities were transformed logarithmically (base 10) to calculate confidence intervals, so as to estimate the population parameter for the mean at each frequency separately for each time series. Spectral densities higher than the upper boundary of the $95 \%$ confidence limit were used to define peaks in the spectrum (10) of the individual time series. This procedure enables us to define spectral density values that are not due to chance $(P<0.05)$ and therefore represent "real" rhythms in the individual time series. 


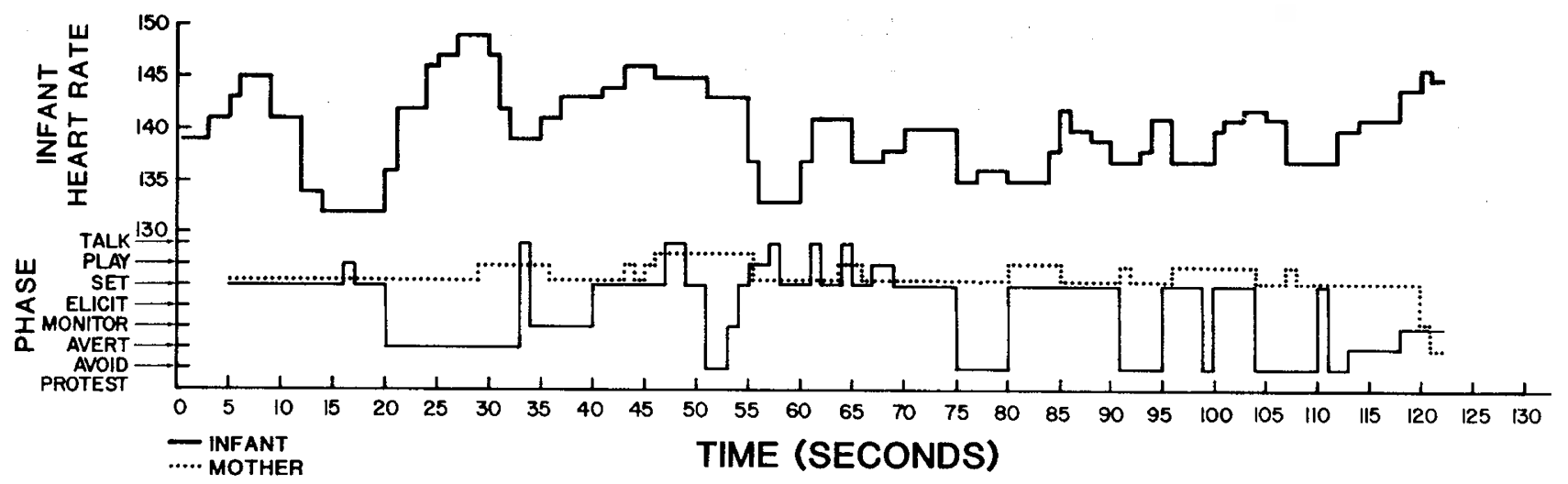

Fig. 1. Maternal and infant behavior (monadic phases) and infant heart rate during social interaction.

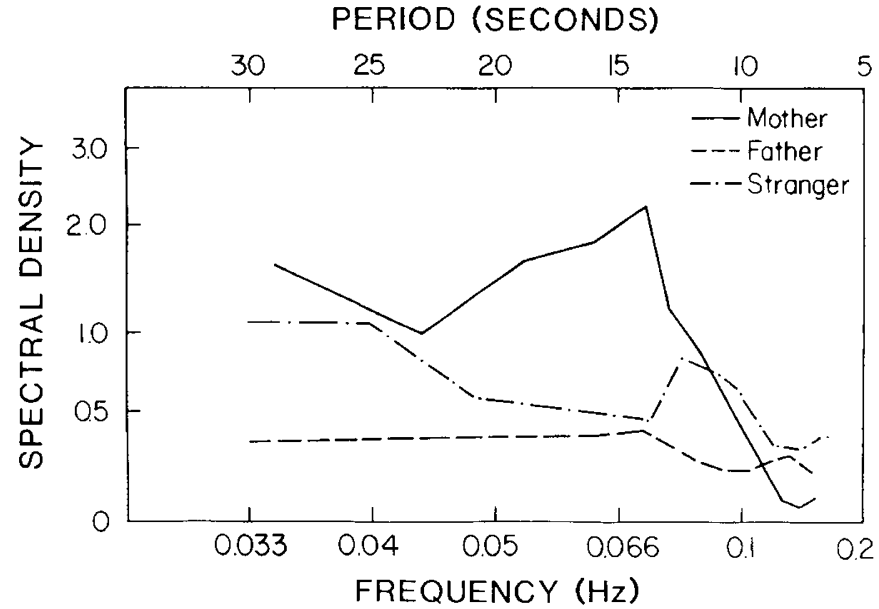

Fig. 2. Spectral density $\left(\log _{10}\right)$ of infant monadic phase with adult interactant.

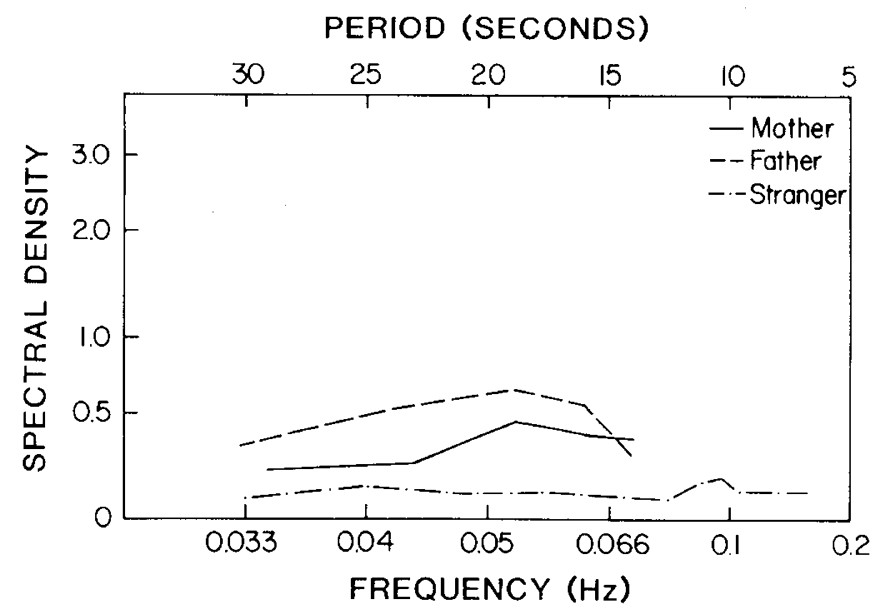

Fig. 3. Spectral density $\left(\log _{10}\right)$ of adult monadic phase with infant.

Spectral peaks in the power spectrum are shown for infant behavior during interaction with mother, father, and stranger in Figure 2. Frequency $(\mathrm{Hz})$ is shown on the lower abscissa and the period (in time) is shown on the upper abscissa. With mother, the infant's behavior showed a major spectral peak in the frequency band around $14 \mathrm{sec}(0.071 \mathrm{~Hz})$. During interaction with the stranger, the highest peaks for infant behavior were in the band from $25-30 \mathrm{sec}(0.033-0.04 \mathrm{~Hz})$. With the father there is less overall power in the spectrum for infant behavior, although the highest peaks occur in the same band of frequencies as with mother. Figure 3 shows the peaks in the power spectrum for adult behavior during interaction with the infant. The spectral

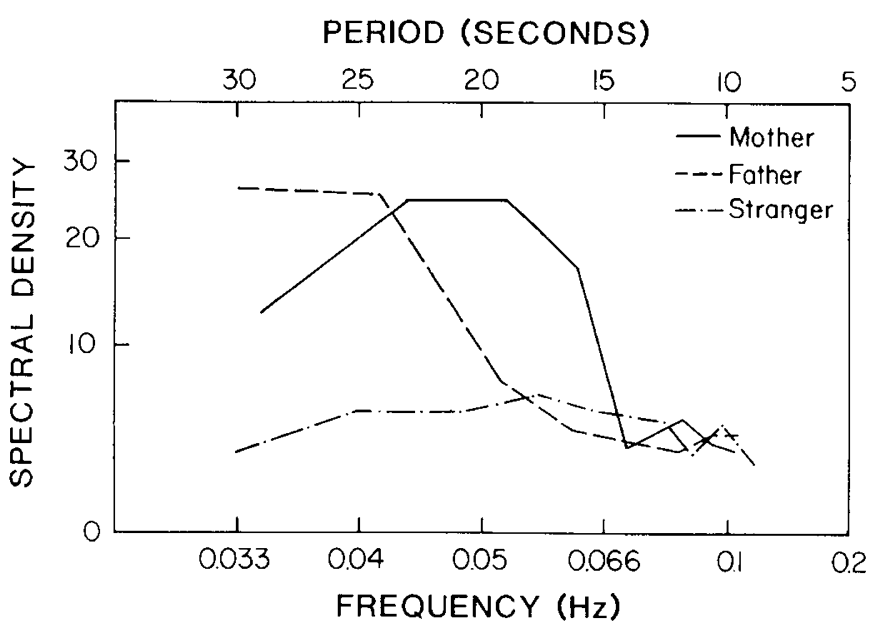

Fig. 4. Spectral density $\left(\log _{10}\right)$ of infant heart rate with adult interactant.

peaks are similar for mother and father and higher for both parents than for the stranger. For the stranger, the spectral peaks appear to be lower overall.

The spectral peaks for infant heart rate during interaction with the three adults are shown in Figure 4. With mother, the infant's heart rate spectrum showed a major peak in the band of frequencies from $19-23 \mathrm{sec}(0.043-0.052 \mathrm{~Hz})$. With father, the infant's heart rate spectrum showed major peaks in the band from 24$30 \mathrm{sec}(0.033-0.041 \mathrm{~Hz})$ with similar spectral density values. By contrast, with the stranger, the infant's heart rate showed peaks whose overall spectral densities were lower than those with either mother or father.

Table 1 shows the coherence values between infant heart rate and behavior and between infant behavior and adult behavior during social interaction. Coherence was only computed at each frequency where both series showed spectral peaks. Peaks in the coherence spectrum were then defined just as for spectral density peaks, using the upper limit of the $95 \%$ confidence interval (10).

As can be seen in Table 1 for the mother-infant dyad, the coherence spectrum for maternal and infant behaviors showed a peak at $23 \mathrm{sec}(0.43 \mathrm{~Hz})$. The coherence between infant heart rate and infant behavior with mother peaked at $11.8 \mathrm{sec}(0.84$ $\mathrm{Hz}$ ).

For the father-infant dyad, peaks in the coherence spectrum for paternal and infant behavior were found at 14 and $30 \mathrm{sec}$ $(0.033$ and $0.071 \mathrm{~Hz})$. The coherence between infant heart rate and infant behavior with father peaked at 24 and $30 \mathrm{sec}(0.033$ and $0.041 \mathrm{~Hz}$ ).

Finally, for the stranger-infant dyad there were no peaks in the coherence spectrum between stranger and infant behavior. The coherence between infant heart rate and infant behavior with stranger peaked at $10.4 \mathrm{sec}(0.096 \mathrm{~Hz})$. 
BEHAVIOR AND HEART RATE

Table 1. Coherence between infant heart rate and behavior and between infant and adult behavior during social interaction'

\begin{tabular}{|c|c|c|c|c|c|c|c|}
\hline \multirow{2}{*}{$\begin{array}{l}\text { Frequency of } \\
\text { peak }(\mathrm{Hz})\end{array}$} & \multirow{2}{*}{$\begin{array}{c}\text { Period } \\
(\mathrm{sec})\end{array}$} & \multicolumn{3}{|c|}{$\begin{array}{l}\text { Coherence between infant heart } \\
\text { rate and infant behavior }\end{array}$} & \multicolumn{3}{|c|}{$\begin{array}{l}\text { Coherence between infant behavior } \\
: \quad \text { and adult behavior }\end{array}$} \\
\hline & & Mother & Father & Stranger & Mother & Father & Stranger \\
\hline 0.033 & 30 & & $0.93^{2}$ & 0.20 & & $0.87^{2}$ & 0.45 \\
\hline 0.034 & 29 & 0.23 & & & 0.19 & & \\
\hline 0.040 & 25 & & & 0.02 & & & 0.19 \\
\hline 0.041 & 24 & & $0.57^{2}$ & & & 0.45 & \\
\hline 0.043 & 23 & 0.12 & & & $0.77^{2}$ & & \\
\hline 0.048 & 20.8 & & & 0.26 & & & 0.33 \\
\hline 0.052 & 19 & 0.19 & 0.10 & & 0.10 & 0.15 & \\
\hline 0.062 & 16 & 0.01 & 0.23 & & 0.18 & 0.07 & \\
\hline 0.071 & 14 & 0.32 & & & 0.13 & $0.53^{2}$ & \\
\hline 0.072 & 13.8 & & & 0.27 & & & \\
\hline 0.08 & 12.5 & & & 0.41 & & & 0.32 \\
\hline 0.083 & 12 & & 0.10 & & & & \\
\hline 0.084 & 11.8 & $0.84^{2}$ & & & & & \\
\hline 0.088 & 11.3 & & & 0.37 & & & 0.47 \\
\hline 0.092 & 10.8 & & 0.06 & & & & \\
\hline 0.096 & 10.4 & & & $0.69^{2}$ & & & 0.29 \\
\hline 0.102 & 9.8 & & 0.29 & & & & \\
\hline 0.112 & 8.9 & & & 0.35 & & & \\
\hline 0.144 & 6.9 & & & & & & 0.31 \\
\hline
\end{tabular}

${ }^{1}$ Coherence computed only at frequencies where both time series showed spectral peaks.

${ }^{2}$ Coherence peak defined as greater than $95 \%$ confidence interval (see text).

\section{DISCUSSION}

Rhythmicity in social interaction and between behavioral and physiologic activity suggests that this infant displays differential cycles of behavioral affect and heart rate while interacting with her mother, father, or stranger. The highest spectral density values, indicating stronger behavioral rhythms in the infant, were found in the presence of the mother. The lower spectral densities of infant behavior with stranger and father may be due to an order effect because stranger and father followed mother and the infant became more fatigued and irritable as the session progressed. It is interesting to note, however, that even though the spectral density values are lower with the father, the highest peak in the spectrum is at the same rhythm as the mother's highest peak, $14 \mathrm{sec}(0.071 \mathrm{~Hz})$. This may indicate that the infant shows different rhythms of affective behavior with mother and father than with stranger.

Cycles of maternal and paternal behavior showed spectral peaks across similar frequency bands and were consistently of higher spectral density values than the spectral peaks in the stranger's affective rhythms. It is likely that previous experience with an infant facilitates the establishment of social interactive rhythms in adults; therefore, the lower level of behavioral cycling in the stranger is not surprising.

Rhythms of the infant's heart rate were stronger (peaks of higher spectral density) with both mother and father than they were with the stranger. This strong rhythmicity was present across a wider band of frequencies with mother (period of 15-30 sec) than with father (a slower frequency period of 25-30 sec), although major peaks exist at overlapping frequencies with both parents. Infant heart rate rhythms with the stranger are of substantially lower density across all frequencies. Once again, the infant's familiarity with her parents seems to facilitate the establishment of strong heart rate rhythms during social play with her parents. With a strange adult, the infant displays more variability in her heart rate patterns, and this is reflected in the lower spectral density of her heart rate rhythms. This methodology illustrates the presence of rhythmicity in both infant behavioral and physiologic systems during social interaction with both parents and a stranger, but also suggests qualitative differences in the strength and/or frequency of the rhythmicity with parents as compared with a stranger.
The coherence between rhythms of adult and infant behavior and of infant heart rate and behavior provides further support for the differing rhythmicity with parents and a stranger. At several different frequencies, the rhythms of mother and infant behavior, $23 \mathrm{sec}(0.043 \mathrm{~Hz})$, and of father and infant behavior, 14 and $30 \mathrm{sec}(0.033$ and $0.071 \mathrm{~Hz})$, were related, whereas the rhythms of stranger and infant behavior were not related. Even though the infant may have been fatigued by the time father interacted and her rhythms were less strong, they still correlate with father's rhythms suggesting that father remains sensitive to the infant's negative behaviors and that father and infant are still able to achieve some synchrony in their interaction.

This supports previous findings that infants are more synchronous during social interaction with mothers and fathers than with strangers. In earlier work, simultaneous behavioral transitions between monadic phases (in which both partners shifted within $2 \mathrm{sec}$ of each other) were used as evidence for synchrony (23) during interaction between mothers/fathers and infants.

The illustration of differences in rhythmic synchrony suggests that this technique may be useful for the study of infant differentiation of familiar parents from unfamiliar strangers. It suggests that the differentiation of strangers by infants may exist by 3 months and may be dependent upon previous patterns of rhythmic social interaction established with familiar adults.

Finally, the coherence spectrum indicates that infant heart rate and behavioral rhythms are associated with all three adult interactants. It is not surprising that in a healthy, well-organized infant, the infant's behavioral and heart rate rhythms are synchronized regardless of the adult interactant. This finding supports the hypothesis that a biologic basis for the rhythmic entrainment of both heart rate and behavior may exist in the young infant. It is also possible that the infant's behavioral and physiologic systems are organized under similar rules of both behavioral and physiologic feedback and control (2).

It is intriguing to note that the synchrony between infant heart rate and behavioral rhythms occurred at different frequencies with mother and stranger (periods of $10-11 \mathrm{sec}$ ) than it did with father (periods of $25-30 \mathrm{sec}$ ). This could be due to the differing physiological arousal related to the infant's negative behavior.

The generalizability of these results to other infants and even to the same infant and adults on different occasions has not yet been established. The methodologic advantage of an approach 
that simultaneously evaluates behavioral and cardiac rhythmicity during social interaction has been demonstrated. Applying the technique of spectral analysis to a single case study of adultinfant social interaction supports the theoretical construct that rhythmicity is a basic ingredient of developing social interactions. Furthermore, it suggests that differences in rhythmicity can be used to study variations in social interaction with different adults. Although the findings from a single case study are preliminary and need replication, for this infant the rhythms for infant heart rate and the coherence between infant and adult behavior during interaction were similar with mother and father and were different with the stranger, providing early evidence of differential interaction. The ability of this technique to distinguish the more synchronous rhythms of the infant with her mother and father from the less synchronous rhythms with the stranger suggests that it may prove clinically useful in distinguishing failing interactions in disorders such as failure to thrive and other parenting disorders, and in describing the disorganized rhythmicity of infants with autism and central nervous system disorders.

This study suggests that human behavioral and physiologic systems may be temporally organized and linked at the level of microrhythms (at least during social interaction), as well as at the level of circadian rhythms.

\section{REFERENCES AND NOTES}

1. Bakeman, R. and Brown, J. V.: Behavioral dialogues: An approach to the assessment of mother-infant interaction. Child Develop. 48:195 (1978)

2. Brazelton, T. B., Koslowski, B., and Main, M.: The origins of reciprocity. In M. Lewis and L. A. Rosenblum (eds.), The Effect of the Infant on its Caregiver, (Wiley, New York: 1974).

3. Brazelton, T. B., Tronick, E., Adamson, L., Als, H., and Wise, S.: Early mother-infant reciprocity. In R. Hinde (ed.), Parent-Infant Inter. (Elsevier, Amsterdam 1975).

4. Bullowa, M.: (ed.) Before Speech. (Cambridge: Cambridge University Press, 1979).

5. Condon, W. S. and Sander, L. W.: Neonate movement is synchronized with adult speech. Science, 183 (4120): 99 (1978).

6. Dixon, S., Yogman, M. W., Tronick, E., Adamson, L., Als, H., and Brazelton, T. B.: Early infant social interaction with parents and strangers. J. Amer. Acad. Child Psychiat., 20: 32 (1981).

7. Field, T.: Interaction behaviors of primary versus secondary caretaker fathers. Develop. Psychol., 14: 181, (1978).

8. Gottman, J. M.: Detecting cyclicity in social interaction. Psychol. Bull., 86 . 338 (1979).
9. Gottman, J. M. and Ringland, J. T.: The analysis of dominance and bidirectionality in social development. Child Develop.. 52: 293 (1981).

10. Jenkins, G. M. \& Watts, J. G.: Spectral Analysis and its Applications. (San Francisco: Holden Day, 1968).

11. Kaye, K. and Fogel, A.: The temporal structure of face-to-face communication between mothers and infants. Develop. Psychol., 16: 454 (1980).

12. Lester, B. M., Hoffman, J., and Brazelton, T. B.: Spectral analysis of motherinfant interaction in term and preterm infants. Paper presented at International Conference on Infant Studies, 1982.

13. Moore-Ede, M., Sulzman, F. M. and Fuller, C. A.: The Clocks That Time Us. p. 166-190 (Harvard University Press, Cambridge 1982).

14. Porges, S., Bohrer, R., Cheung. M., Drasgow, F., McCabe, P., and Karen, G. New time-series statistic for detecting rhythmic co-occurrence in the frequency domain: the weighted coherence and its application to psychophysiological research. Psychological Bult., 87: 580 (1980).

15. Sander, L. W., Julia, H., Stechler, G., Burns, P., and Gould, J.: Some determinants of temporal organization in the ecological niche of the newborn. Paper presented at the International Society for the Study of Behavioral Development, 1975.

16. Sander, L., Stechler, G. Burns, P., and Lee, A. Changes in infant and caregiver variables over the first two months of life: Integration of action in early development. In E. B. Thoman (ed.) Origins of the Infant's Social Responsiveness. (Hillsdale, NJ: Erlbaum, 1979).

17. Statistical Analysis System. p. 281 (SAS institute, Raleigh, 1979)

18. Stern, D. N.: The goal and structure of mother-infant play. J Amer. Acad. Child Psychiat., 13: 402 (1974)

19. Stern, D. and Gibbon, J.: Temporal expectancies of social behaviors in motherinfant play. In E. B. Thoman (ed.), Origins of the Infant's Social Responsiveness. (Erlbaum, Hillsdale, 1979).

20. Tronick, E.: The structure of face-to-face interaction and its developmental functions. Paper presented at the Society for Research in Child Development, 1977.

21. Tronick, E., Als, H., \& Brazelton, T. B.: Monadic phases: a structural descriptive analysis of infant-mother face-to-face interaction. Merrill-Palmer Quarterly, 26: 3 (1980).

22. Yogman, M.: The goals and structure of face-to-face interaction between infants and fathers. Paper presented to the Society for Research in Child Development. 1977.

23. Yogman, M.: Development of the father-infant relationship. In: H. Fitzgerald B. Lester, and M. Yogman (eds.) Theory and Research in Behaviora Pediatrics. Vol. 1 (Plenum Press: New York, 1982).

24. This research was part of a larger project done in collaboration with Dr. Suzanne Dixon and with the advice and assistance of Dr. T. B. Brazelton and Ed Tronick, Ph.D. The research was supported by the Robert Wood Johnson Foundation, the Carnegie Corporation of New York, and the National Foundation March of Dimes.

25. Requests for reprints should be addressed to: Dr. Michael W. Yogman, Associate Chief, Division of Child Development, Children's Hospital Medical Center, 300 Longwood Avenue. Boston, Massachusetts 02115.

26. Received for publication April 22, 1982.

27. Accepted for publication March 21, 1983. 